\title{
WSN Routing Algorithm Used in Large Water Area Based on PEGASIS
}

\author{
Jie-Ping Yu, Guo-Hui Jiao \\ College of Electronic Engineering, South China \\ Agricultural University, China \\ E-mail: 489398737@qq.com, 10353907779@qq.com
}

\author{
Wei-Xing Wang, Sheng Jiang, Hui-Li Yin \\ College of Electronic Engineering, South China \\ Agricultural University, China \\ Guangdong Engineering Research Center for Monitoring \\ Agricultural Information, China \\ Key Laboratory of Information Acquisition and \\ Application in Agriculture, Guangzhou Science \\ Technology and Innovation Commission, China \\ E-mail: weixing@scau.edu.cn,410252413@qq.com, \\ 76576676@qq.com
}

\begin{abstract}
With a view to the nodes deployment of the water quality on-line monitoring system based on WSN, and the problem of the imbalanced node energy consumption caused by the unreasonable selection of the chain tag in PEGASIS agreement, this paper puts forward a routing algorithm of optimal energy heterogeneous adaptive link(OEHAL) suitable for large water area monitoring. On the basis of the residual energy of nodes and the distance between the node and the base station, the algorithm selects the head node of the chain tag, so as to achieve the balance of energy consumption. The redesign of the optimal link prolongs the life cycle of the nodes and reduces the time delay of communication. Matlab2014b software is used to simulate the algorithm, and the results show that the network performance of the algorithm is better than PEGASIS algorithm and is practical in the water quality monitoring network of large water area specially.
\end{abstract}

Keywords-WSN; routing algorithm; PEGASIS

\section{INTRODUCTION}

With the characters of low cost, low power consumption and high reliability etc, WSN, Wireless Sensor Network, has very extensive application in modern times[1-5].

Its application areas include homeland security, monitoring of surface water and soil, the defense intelligence collection, environmental monitoring, analysis and forecast of weather and climate. The environmental awareness, which is distributed and based on the simple and flexible deployment of wireless communication technology, makes WSN become the important factors that affect our daily life.

Because of the limited energy of wireless sensor network node, the most energy-efficient data transmission is needed, and at the same time the accuracy of the information cannot be reduced. Therefore, the problems of node power consumption should be considered first when researching the WSN routing protocol. On the premise of guarantee stable data transmission, the network lifetime will be prolonged as much as possible.

In large monitoring areawireless sensor networks often encounter a problem that some nodes will die early because of long transmission distance which will affect the stability of network nodes, especially in the water area. Due to the refraction scattering of the signal caused by fluctuation of water surface, signal interference stronger.

\section{RESEARCH STATUS}

Regarded the communication way the nodes involved, wireless sensor network routing protocol can be divided into planar routing protocol and hierarchical routing protocol. Planar routing protocol mainly includes SPIN, Sensor separate Protocols for Information via Negotiation, and DD, Directed coursing, etc. But due to the large number of nodes in wireless sensor networks, planar routing protocol will cause large network spending and limits the network lifetime. Hierarchical routing protocol mainly includes LEACH, Low Energy Adaptive Clustering Hierarchy, PEGASIS, PowerEfficient Gathering in Sensor Information Systems and Threshold sensitive Energy Efficient sensor Network protocol, TEEN, etc.

The purpose of LEACH agreement[6] is to select sensor nodes in a dynamic network as the cluster head nodes and to form a cluster. The sensed data within cluster nodes are directed to the cluster head nodes and data fusion. Cluster head nodes transfer the collected data to base station directly.

PEGASIS[7] is a chained protocol published by $\mathrm{S}$. Lindsey and others based on LEACH. It developed from LEACH, but it is very different from the topology of LEACH for that LEACH is cluster structure, and PEGASIS is chain structure. And by greedy Algorithm, in PEGASIS, all nodes in the network will be completed a link, the collected information will be passed to the head nodes selected by the base station by the link chain, then transferred by chain head nodes to base station.

The formation stage of link begins in the node farthest from the base station. The node sends test signals, by monitoring the strength of the signal other nodes response to determine the nearest node, and adds it to the link. After that, the added node determines the nearest node (not including the node in the link) and add it to the link according to the above method. So on the link is formed including all nodes finally.

After the chain tag node is selected by the base station, the data will be transferred by control mechanism in token type. First, the token signal is passed to two ends of the chain, 
and then send the data to the next node in the chain. After receiving the data, the nodes will fuse its own data and received data and send to the next node. Finally, the data of the two ends of the chain head node is fused and sent to the base station. When there is a node dead in the chain, the network will need to re-establish a chain. Compared with LEACH, the PEGASIS protocol can improve the energy efficiency in a certain extent, but it has a certain degree of communication delay due to the characteristics of sequential transmission. Whats more, it is easy to form long chain, resulting in some problems for example, energy consumption of nodes is excessive and unbalanced, long chain nodes will die soon.

Previous studies have done a lot of researches on improving the PEGASIS protocol. Yu Yongchang and others[8] proposed EECB protocol based on PEGASIS protocol. This protocol considers the residual energy of the node and the distance between the node and the base station in the selection of the chain, and avoids the emergence of long chain through the threshold, which effectively prolongs the lifetime of the network. In combination with the LEACH protocol and PEGASIS protocol, Li Jianqi and others[9] propose an improved protocol, which is the same as the LEACH protocol in the selection of cluster heads and cluster. In the cluster communication, the combination of single hop direct communication and chain communication is adopted. The communication between clusters adopts the chain communication mode, which solves the problem of communication delay in PEGASIS protocol, but does not solve the problem of long chain. Wang Weixing and others[10] proposed the EHMCA protocol, which layers all nodes. In each layer, all nodes form clusters and cluster heads will fuse data. A chain is formed between the cluster head nodes of each cluster according to the PEGASIS protocol, and finally the data converges to the base station. The protocol can effectively reduce the communication delay and increase the life cycle. But there is still the problem of node power consumption is not balanced, resulting in many nodes premature death.

\section{NETWORK AND RADIO MODEL}

Aiming at the problem in PEGASIS protocol that the energy consumption of the chain tag is too fast and easy to die prematurely, which leads to the uneven energy consumption of each node in the network and the problem of long chain, in the energy heterogeneous network, the selection method of the chain tag is revised based on the idea of EECB protocol that we should consider the residual energy of the nodes and the distance between the chain tag and the base station when selecting the chain tag. At the same time, the method of establishing chain is modified, and a routing algorithm of optimal energy heterogeneous adaptive link is proposed. The algorithm selects the optimal chain, therefore, it can avoid the emergence of long chain, so as to effectively slow down the speed of energy consumption. Energy surplus node is selected as the chain tag, which can balance the energy consumption of nodes in the network, and make the network more stable.

\section{A. Network Hypothesis}

The assumption that the network is composed of $\mathrm{N}$ randomly deployed sensor nodes in the $\mathrm{M} * \mathrm{M}$ region, which periodically collect data, and the wireless sensor network has the following characteristics:

- The nodes of the network are distributed randomly and fixedly in the monitoring area;

- There is only one energy abundant base station in the network, which is fixed and far away from the monitoring area

- In the network, the energy of nodes is heterogeneous, and the nodes have the function of data fusion. Each node is assigned a unique ID number and can sense its own energy information and position information;

- In addition to the energy of nodes in the network in the hardware conditions should be the same, and the node energy is limited;

- The transmission power of the nodes in the network is controllable;

- The wireless channel is symmetrical in the network.

\section{B. Algorithm Flow}

Firstly, choose the chain head. Because the node distribution is large, and the location of the base station is far from the distribution range of the common nodes, the chain head always consumes more energy and is easy to die earlier. Therefore, we should consider the residual energy of the nodes and the distance between the node and the base station in the selection of the chain. Each node is to calculate the remaining energy assuming that they become the chain head to complete the current round of data transmission. By comparison, we should select the node with the most residual energy as the chain head.

Secondly, establish the chain. Because of the greedy algorithm, the PEGASIS protocol is easy to generate long chain and long communication delay. In the establishment of the chain, improved algorithm joins the chain head into the chain firstly, then adds an extra node (Node B) with the closest distance to a certain node (Node A) in the chain to the chain and record the nodes' routing relationship. It means that the next hop of the B node is the A node. Such a tree structure chain will eventually converge at the chain node. Finally, data will be sent to the base station through the chain head. As shown in Figure 1.

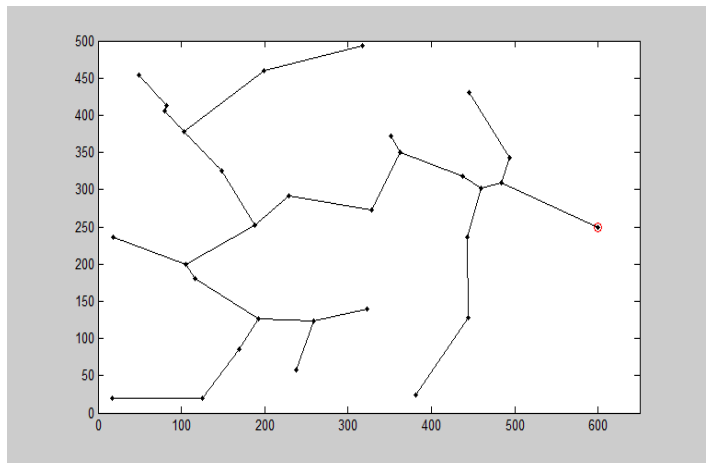

Figure 1. Link structure diagram. 
Compared to the PEGASIS protocol, multiple loop search to establish the chain is used for a longer time and takes up relatively more resources. If the data transmission in the last round of the link does not cause the death of the node, the network does not need to be re-chained.

Just change the direction of the next hop between the new chain tag and the node of the previous one, then the new chain tag would be transferred to the base station directly and we can reuse the last round of the link..

\section{Algorithm Analysis}

The assumption that the network consists of 100 sensor nodes randomly deployed in the $500 * 500$ region. The energy of nodes in the network is heterogeneous, and the base station is far away from the network area and has sufficient energy. According to the distance between the transmitter and the receiver, the different energy consumption models can be used in WSN:

$$
\mathrm{E}_{\mathrm{tx}}(L, d)=\left\{\begin{array}{l}
L E_{t x}+L \varepsilon_{f s} d^{2}, d<d_{0} \\
L E_{t x}+L \varepsilon_{m p} d^{4}, d \geq d_{0}
\end{array}\right.
$$

In the formula 1 , the $E_{t x}$ represents the energy consumed by the node sending $L$ bits of data to the location of the distance $d$, which consists of energy loss of the transmission circuit $\left(\varepsilon_{f_{s}}\right)$ and energy loss of power amplifying $\left(\varepsilon_{m p}\right)$. Free space propagation model is used if the transmission distance $d<d_{0}$, or multi path attenuation model is chosen. $d_{0}$ is the critical value of the two models when the energy consumption is equal. By using formula 1 , the energy consumption of nodes increases with the increase of transmission distance. According to the PEGASIS protocol, each node is to calculate the remaining energy assuming that they become the chain head. That is

$$
\mathrm{E}_{\Delta}(n)=\left(L E_{t x}+L \varepsilon_{m p} d^{4}\right)+2 L E_{t x}
$$

We should select the node with maximum value as the chain tag in this communication after the value of the residual energy of each node minus $\mathrm{E}_{\Delta}(n)$.

\section{Simulation and Analysis}

In order to verify the effectiveness of OEHAL and its superiority in heterogeneous networks, we use the PEGASIS algorithms respectively for simulation and compare the following performance indicators: Network life cycle, which refers to the time period from the beginning to the death of sensor nodes; data transfer quantity; Network life, refers to the duration that the node in the network began to gather information until the network cannot provide the required data to the observer; stable period, which refers to the time when the WSN node begins to collect data until the first node dies.
Table I the parameters used in simulations

\begin{tabular}{ll}
\hline parameter & value \\
\hline network coverage area $(m)$ & $(0,0) \sim(500,500)$ \\
base station location $(m)$ & $(600,250)$ \\
number of nodes & 100 \\
Initial energy of node $\left(\boldsymbol{E}_{\mathrm{O}} / \boldsymbol{J}\right)$ & 0.5 \\
$\mathbf{E}_{\text {elect }}(\boldsymbol{n J} / \boldsymbol{b})$ & 50 \\
$\boldsymbol{E}_{f s}$ & 10 \\
$\boldsymbol{E}_{m p}$ & 0.0013 \\
communication distance $(m)$ & 87.7 \\
$\mathbf{E}_{\boldsymbol{D}}(\boldsymbol{n} \boldsymbol{J} / \boldsymbol{b})$ & 5 \\
packet size $(b)$ & 4000 \\
energy isomer ratio & 1 \\
heterogeneous node ratio & 0.1 \\
\hline
\end{tabular}

Figure 2 shows the comparison of the network node survival cycle. With the PEGASIS protocol, EECB protocol and EHMCA protocol the nodes in network from the beginning of the transmission until all the nodes die, gradually die, so the energy consumption of nodes in the network is not balanced, the network has been in a state of instability. However, the network has been in steady state in nearly 700 rounds according to OEHAL agreement. Node energy consumption is very balanced, and the stability period is greatly improved compared to the PEGASIS protocol. The stable period occupies $76.5 \%$ of the whole network lifetime, which is greatly improved compared to other protocols. Figure 3 is the comparison of the total amount of packets received by the base station. From Figure 3, we can see that in a network life cycle, the total amount of packets received by the base station with OEHAL protocol is about 3.6 times of the network with the PEGASIS protocol, is about 2.7 times of the network with the EECB protocol and is about 1.4 times of the network with the EHMCA protocol. The above analysis shows that the OEHAL protocol can solve the problem of the network energy consumption better in a large area, which makes the whole network more stable.

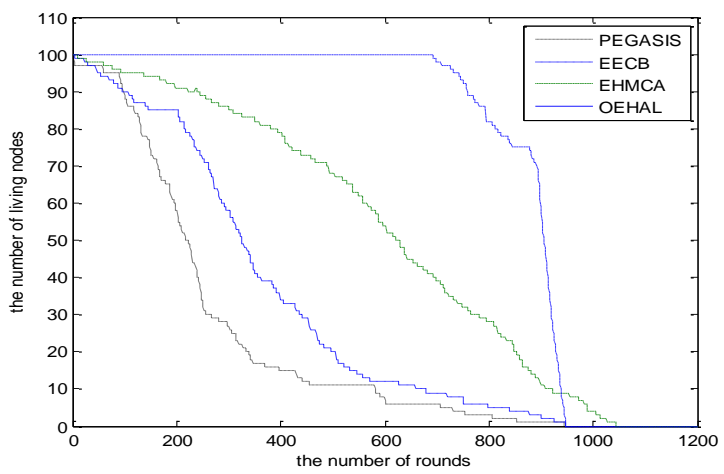

Figure 2. Comparison of network node lifetime. 


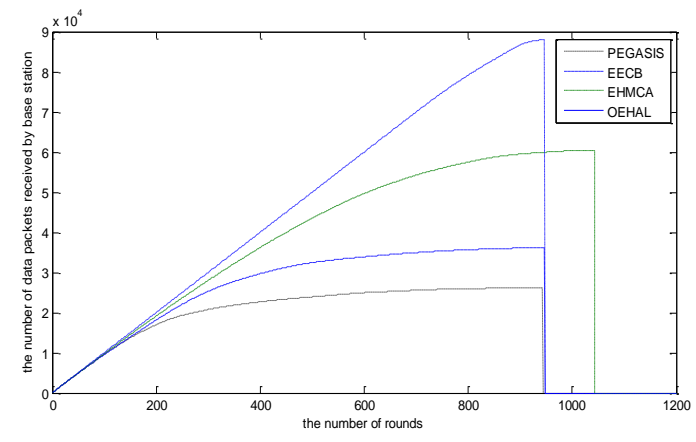

Figure 3. Comparison of data packets received by base station.

\section{CONCLUSION AND DISCUSSION}

Two problems were found in PEGASIS algorithm after analyzing it. One is that it would cause communication latency easily and the other is that the long chain would be formed easily during formation of the link, which would cause early death at both the ends of the long chain nodes.

In view of these two problems, this paper proposes a new improved the OEHAL protocol. The protocol uses a chain structure which is similar to the tree, effectively reduces the communication latency and the problem of forming a long chain, and selects the head of chain through the residual energy and location information of the distance to the base station, which would increase the life cycle of the node in the network sharply, and balance the energy consumption among all the nodes of the network.

Due to some computation overhead caused by multiplesearching adopted by the OEHAL protocol in the link formation, in subsequent studies, a further modified OEHAL protocol is needed to simply realize the computing formed by network link. At the same time, solving the problem on the influence of the water environment on the node data transmission should be focused on to achieve the water quality on-line monitoring used in the environment of large area water.

\section{ACKNOWLEDGMENT}

This research was financially supported by Science and Technology Planning Project of Guangdong Province, China (2014-17)

\section{REFERENCES}

[1] J.M. Kim, H.S. Seo and J. Kwak. Routing Protocol for Heterogeneous Hierarchical Wireless Multimedia Sensor Networks[J]. Wireless Personal Communications.2011. 60(3): 559-569.

[2] J. Yu, et al. A cluster-based routing protocol for wireless sensor networks with nonuniform node distribution[J]. International Journal of Electronics and Communications. 2012. 66(1): 54-61.

[3] Z. Yu, et al. A Reliable Energy-Efficient Multi-Level Routing Algorithm for Wireless Sensor Networks Using Fuzzy Petri Nets[J]. Sensors. 2011. 11(3): 3381-3400.

[4] A.M. Khedr and W. Osamy. Effective target tracking mechanism in a self-organizing wireless sensor network[J]. Journal of Parallel and Distributed Computing. 2011. 71(10): 1318-1326.

[5] R.A.F. Mini and A.A.F. Loureiro. Energy-efficient design of wireless sensor networks based on finite energy budget[J]. Computer Communications, 2012. 35(14): 1736-1748.

[6] W.R. Heinzelman, A. Chandrakasan and H. Balakrishnan. Energyefficient communication protocol for wireless microsensor networks[C]. In Proceedings of the IEEE Hawaii International Conference on System Sciences, pp. 1- 10, Maui, HI, USA, 2000.

[7] S. Lmdsey, C.S. Raghavendra. PEGASIS: Power-Efficient Gathering in Sensor Information Systems[C]. In Proceedings of the IEEE Aerospace Conference Proceedings, Big Sky, MT, USA, 2002.

[8] Yu, Y. and Y. Song. An Energy-Efficient Chain-Based Routing Protocol in Wireless Sensor Network. 2010, International Conference on Computer Application and System Modeling. p. 486-489.

[9] Li Jianqi, et al. Improved Routing Protocol in Wireless Sensor Network Based on LEACH and PEGASIS. Chinese Journal of Sensors and Actuators. 2012. 25(2): 263-267.

[10] Wang Weixing et al. Energy Heterogeneity-Based Multi-Chain Algorithm for WSN. Journal of South China University of Technology (Natural Science Edition). 2015. 43(9): 74-80. 